\title{
Controle Extremal para Mapeamentos Dinâmicos com Sinal de Hessiana Desconhecido
}

\author{
Aline Lopes Dibo* Tiago Roux Oliveira* \\ * Departamento de Engenharia Eletrônica e Telecomunicações \\ Universidade do Estado do Rio de Janeiro (UERJ) \\ Rio de Janeiro, RJ 20550-900, Brasil. (e-mail: alineldibo@gmail.com, \\ tiagoroux@uerj.br)
}

\begin{abstract}
Extremum seeking control aims at determining and keeping the output of a nonlinear map on its unknown extremum point. This paper proposes the process of extremum seeking occurs independent of the Hessian sign information. The key idea is to combine the classical extremum seeking approach with a switching monitoring function. The switching algorithm will drive the closed-loop system to the unknown extremum, neglecting if it is a maximum or a minimum. Unlike our previous results in this topic, where only static maps were considered, we assume dynamic nonlinear maps now. In this sense, the monitoring function must be totally reformulated in order to take into account the general nonlinear dynamics derived from a singular perturbation analysis. Local exponential stability and convergence to an arbitrarily small neighborhood of the extremum point are rigorously guaranteed without the knowledge of the Hessian signs. Numerical simulations show the efficiency of the proposed extremum seeking method in solving sequential real-time optimization problems of maximization and minimization.

Resumo: O controle extremal tem como objetivo determinar e manter a saída de um mapeamento não-linear desconhecido em seu ponto de extremo. Neste trabalho, propõe-se que o processo de busca pelo extremo (busca extremal) se dê independentemente da informação sobre o sinal da Hessiana. A ideia chave para que o processo de otimização aconteça é combinar o esquema clássico de controle extremal com uma função de monitoração chaveada. O algoritmo de chaveamento irá conduzir o sistema em malha fechada ao extremo desconhecido, independente de se tratar de um ponto de máximo ou de mínimo. Ao contrário de nossos resultados anteriores onde apenas mapas estáticos foram considerados, assumimos agora mapas dinâmicos não lineares. Desta forma, a função de monitoração deve ser totalmente reformulada para que se leve em conta a dinâmica não-linear geral que deriva de uma análise de perturbação singular. A estabilidade exponencial local e a convergência para uma vizinhança arbitrariamente pequena do ponto extremo são rigorosamente garantidas sem o conhecimento dos sinais da Hessiana. Simulações numéricas mostram a eficiência do método de busca extremal proposta na solução de problemas sequenciais de otimização em tempo real de maximização e minimização.
\end{abstract}

Keywords: Extremum-seeking Control, Adaptive Control, Uncertain Nonlinear Systems, Switching Monitoring Function, Real-time Optimization.

Palavras-chaves: Controle Extremal, Controle Adaptativo, Sistemas Não-Lineares Incertos, Funções de Monitoração Chaveadas, Otimização em Tempo Real.

\section{INTRODUÇÃO}

O controle extremal, ou busca extremal, do inglês extremum seeking control (ESC) surgiu com (Leblanc, 1922), e se desenvolveu intensamente entre 1940 e 1970 (Aminde, 2013). Mas foi recentemente que o controle extremal atingiu sua maior popularidade (Tan et al., 2010), obtendo resultados promissores para as mais diversas aplicações (Krstic et al., 2014), sendo considerada uma das áreas mais promissoras do controle adaptativo (Astrom and Wittenmark, 2005).

\footnotetext{
* Este trabalho foi financiado pela CAPES, CNPq e FAPERJ.
}

O controle extremal é um método de otimização em tempo real que não se baseia no conhecimento de modelos (Krstic and Wang, 2000). O método tem como objetivo determinar o extremo de uma função quando aplicado em situações em que se depara com uma não-linearidade, e esta apresenta um mínimo ou máximo no problema de controle. Essa nãolinearidade pode ser física, estar inserida na planta, ou pode estar ainda na formulação do problema, por meio de uma função objetivo de um problema de otimização (Zhang and Ordonez, 2012).

Recentemente, a área do ESC alcançou uma certa maturidade e uma nova geração de estratégias de ESC foi aplicada a uma ampla classe de problemas, tais como: 
busca extremal para sistemas dinâmicos híbridos (Poveda and Teel, 2017), sistemas dinâmicos com atrasos (Oliveira et al., 2017), equações diferenciais parciais (Feiling et al., 2018) e busca extremal estocástica (Liu and Krstic, 2012), para citar alguns.

No geral, em um problema de otimização clássica assumese que o sinal da Hessiana é sempre conhecido. Em (Dibo and Oliveira, 2018) e (Dibo and Oliveira, 2019) questionou-se a necessidade do conhecimento desse sinal e proposto que a otimização de um mapeamento estático fosse feita sem que se pudesse afirmar que o ponto de extremo é um máximo ou um mínimo. Contornou-se essa falta de conhecimento utilizando um algoritmo de chaveamento baseado em uma função de monitoração e com isso foi possível obter a estimativa do gradiente.

A maior parte dos trabalhos utilizando controle extremal consideram a planta como um mapa estático. Porém, algumas referências abordam problemas em que a planta dinâmica descrita por uma equação diferencial ordinária está em cascata com o mapa estático não linear. Isso acontece, por exemplo, em (Krstic and Wang, 2000). A proposta deste artigo é generalizar o método aplicado em (Dibo and Oliveira, 2018) e (Dibo and Oliveira, 2019), considerando desta vez uma planta dinâmica apresentado em (Krstic and Wang, 2000).

\section{FORMULAÇÃO DO PROBLEMA}

Em (Krstic and Wang, 2000), é considerada a seguinte planta como um modelo não-linear SISO

$$
\begin{aligned}
& \dot{x}=f(t, u), \\
& y=h(x),
\end{aligned}
$$

sendo $x \in \mathbb{R}^{n}$ o estado, $u \in \mathbb{R}$ a entrada, $y \in \mathbb{R}$ a saída, $f: \mathbb{R}^{n} \times \mathbb{R} \rightarrow \mathbb{R}^{n}$ e $h: \mathbb{R}^{n} \rightarrow \mathbb{R}$. Supondo que a lei de controle seja dada por:

$$
u=\alpha(x, \theta),
$$

parametrizada por um parâmetro escalar $\theta$. Por simplicidade, os autores assumem que se conhece a lei de controle de realimentação de estado estático, com isso seria trivial estender os resultados para a saída dinâmica em malha fechada. É assumido também por simplicidade que $\theta$ e $y$ são escalares. Tem-se, então, o seguinte sistema em malha fechada:

$$
\dot{x}=f(t, \alpha(x, \theta)),
$$

com equilíbrio parametrizado em $\theta$.

A partir deste cenário, chega-se a um mapa estático quadrático de uma única entrada.

Para utilizar o método visto em (Dibo and Oliveira, 2018) e (Dibo and Oliveira, 2019) também em mapeamentos dinâmicos, assumimos que em (Krstic and Wang, 2000) o sinal da Hessiana é desconhecido. A principal consideração a ser feita no estudo utilizado como base é em relação a Hipótese A.2 do artigo referência reproduzido no Apêndice, que para essa suposição, pode ser reescrita da seguinte forma:

Hipótese 2.1: Existe $\theta^{*} \in \mathbb{R}$ tal que:

$$
\begin{gathered}
(h \circ l)^{\prime}\left(\theta^{*}\right)=0, \\
(h \circ l)^{\prime \prime}\left(\theta^{*}\right) \neq 0 .
\end{gathered}
$$

Essa hipótese é diferente do que é comumente visto na literatura, mas isso se deve ao fato de que está sendo considerado que a Hessiana é desconhecida. Sendo a Hessiana, a segunda derivada da saída do mapeamento estático ou função custo com respeito a sua entrada, temos que $(h \circ l)^{\prime \prime}\left(\theta^{*}\right) \neq 0$ e $\left(\theta^{*}, y^{*}\right)$ é o valor extremo desconhecido $\operatorname{com} \theta^{*} \in \mathbb{R}$ e $y^{*} \in \mathbb{R}$.

Assim como em (Dibo and Oliveira, 2018), o problema da falta de conhecimento do sinal da Hessiana será contornado utilizando um algoritmo de chaveamento baseado em uma função de monitoração para a estimativa do gradiente. Com isso, o esquema de busca extremal para sistema dinâmico, visto em (Krstic and Wang, 2000), pode ser redesenhado conforme visto na Figura 1.

Na Figura 1, nota-se a presença da entrada $\theta$ e do ponto desconhecido que otimiza o mapa, $\theta^{*}$, além de um filtro passa-alta $s /\left(s+\omega_{h}\right)$ e um filtro passa-baixa $\omega_{l} /\left(s+\omega_{l}\right)$. Pode ser visto também, a função de monitoração $\varphi_{m}(t)$, onde $k \in[0,1, \ldots]$ é o índice de chaveamento gerado pela função de monitoração e $m \in\{0,1\}$. Logo, dependendo da escolha de $m$ (0 ou 1) podemos definir o valor inicial para a estimativa da direção de controle desconhecida $(-1)^{(k+m)}$ (Rodrigues and Oliveira, 2017). Ainda por meio da Figura 1, define-se facilmente o sinal de controle $U(t)$ por:

$$
U(t)=(-1)^{(k+m)} k_{i} \xi(t)
$$

Tanto a análise do esquema, visto na Figura 1, quanto a seleção de parâmetros são intrigantes. Esses parâmentros são definidos da seguinte forma:

$$
\begin{aligned}
\omega_{h} & =\omega \omega_{H}=\omega \delta \omega_{H}^{\prime}=\mathcal{O}(\omega \delta) \\
\omega_{l} & =\omega \omega_{L}=\omega \delta \omega_{L}^{\prime}=\mathcal{O}(\omega \delta) \\
k_{i} & =\omega K=\omega \delta K^{\prime}=\mathcal{O}(\omega \delta)
\end{aligned}
$$

sendo $\omega$ e $\delta$ constantes positivas pequenas e $\omega_{H}^{\prime}, \omega_{L}^{\prime}$ e $K^{\prime}$ são constantes positivas de $\mathcal{O}(1)$ (Krstic and Wang, 2000).

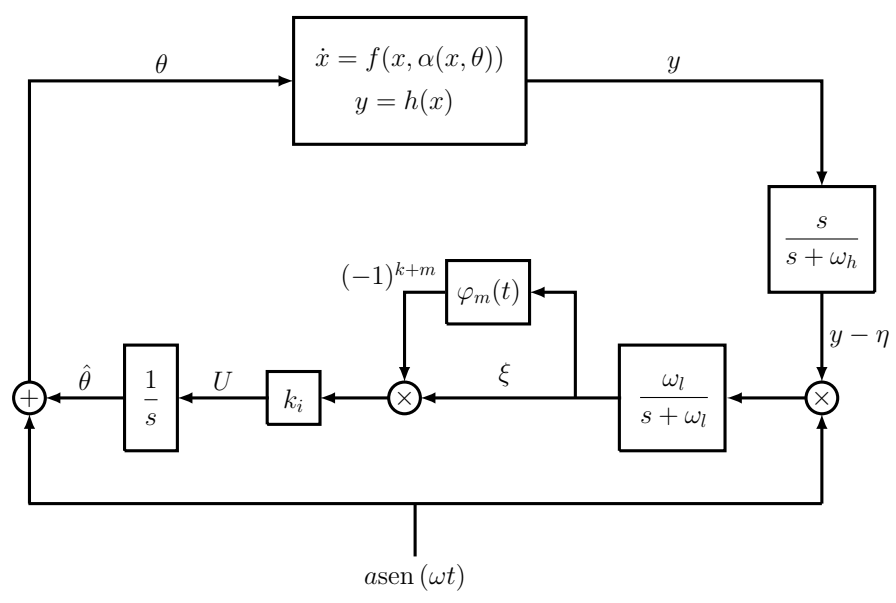

Figura 1. Esquema de busca extremal para sistema dinâmico com sinal da Hessiana desconhecido.

\section{MAJORANTE DO GRADIENTE}

A alternativa proposta para contornar a falta de conhecimento do sinal da Hessiana é a função de monitoração. sendo esta construída com base no majorante do Gradiente (Dibo and Oliveira, 2018). 
Para encontrar a equação do majorante, vamos tomar como referência o Teorema A.1, visto no Apêndice. Esse resultado, apresentado por (Krstic and Wang, 2000), afirma que a solução de $\xi(t)$ converge exponencialmente para uma vizinhança de $\mathcal{O}(\omega+\delta+a)$. Com isso, podemos escrever o majorante de $\xi(t)$ como sendo

$$
|\xi(t)| \leq e^{-\lambda_{1}\left(t-t_{i}\right)}\left|\xi\left(t_{i}\right)\right|+R e^{-\lambda_{2} t}+\mathcal{O}(\omega+\delta+a),
$$

sendo $\lambda_{1}>0$ uma constante de $\mathcal{O}(\omega+\delta+a)$, e $t_{i}$ um instante de tempo inicial arbitrário, enquanto que $\lambda_{2}>0$ e $R>0$ são constantes desconhecidas. O segundo termo da inequação (9), é uma exponencial desconhecida com $R$ dependendo das condições iniciais $(x(0), \hat{\theta}(0), \xi(0), \eta(0))$ do Teorema A.1 do artigo referência.

\section{PROJETO DA FUNÇÃO DE MONITORAÇÃO}

Como dito anteriormente, o projeto da monitoração se dá a partir do majorante do Gradiente. Definimos então a função auxiliar $\varphi_{k}(t)$ como

$$
\begin{aligned}
\varphi_{k}(t)= & e^{-\lambda\left(t-t_{k}\right)}\left|\xi\left(t_{k}\right)\right|+a(k) e^{-\frac{t}{a(k)}}+ \\
& a(k)(\omega+\delta+a),
\end{aligned}
$$

onde $\lambda>0$ é uma constante de projeto de $\mathcal{O}(\omega+\delta+a)$, o termo $t_{k}$ é o instante em que ocorre o chaveamento, $k$ é a quantidade de chaveamentos e $a(k)$ é qualquer sequência monotonicamente crescente em $k$.

A função de monitoração $\varphi_{m}(t)$ pode ser definida como (Oliveira et al., 2007)

$$
\varphi_{m}(t)=\varphi_{k}(t), \quad \forall \in\left[t_{k}, t_{k+1}\right)(\subset[0,+\infty)) .
$$

Como estamos tratando de uma abordagem em que se desconhece o sinal da Hessiana, é impossível fazer qualquer afirmação sobre o extremo desse sistema. Então, invoca-se $\varphi_{m}$ como majorante para a estimativa do gradiente $\xi(t)$. A partir de (11), sempre $|\xi(t)| \leq \varphi_{k}\left(t_{k}\right)$ em $t=t_{k}$. Deste modo, o tempo de chaveamento $t_{k}$ será definido (para $k \geq 0$ ) por

$$
t_{k+1}=\left\{\begin{array}{l}
\min \left\{t \geq t_{k}:|\xi(t)|=\varphi_{k}(t)\right\} \\
+\infty
\end{array}\right.
$$

onde $k \in[1,2, \ldots]$ e $t_{0}:=0$. Por construção, a seguinte desigualdade é obtida de (11)

$$
|\xi(t)| \leq \varphi_{m}(t), \quad \forall t \in[0,+\infty) .
$$

A função de monitoração pode ser vista como um operador híbrido (Goebel et al., 2012) onde cada salto é definido pelo tempo de chaveamento $t_{k}$ (12). Após cada salto, um decaimento de forma exponencial é percebido, esse comportamento é chamado de fluxo, e a Figura 2 ilustra o valor absoluto do gradiente ao longo do tempo e a função de monitoração (Rodrigues and Oliveira, 2017), a última sendo levada à convergência quando a primeira alcança também esta condição (Dibo and Oliveira, 2018)

\section{ANÁLISE DE ESTABILIDADE}

Teorema 5.1: Considere a planta (1) e (2), com a lei de controle (3) e a função de monitoração (11). Então, se $\omega$ na perturbação senoidal $a$ sen $\omega t$ é suficientemente grande, pode-se concluir que

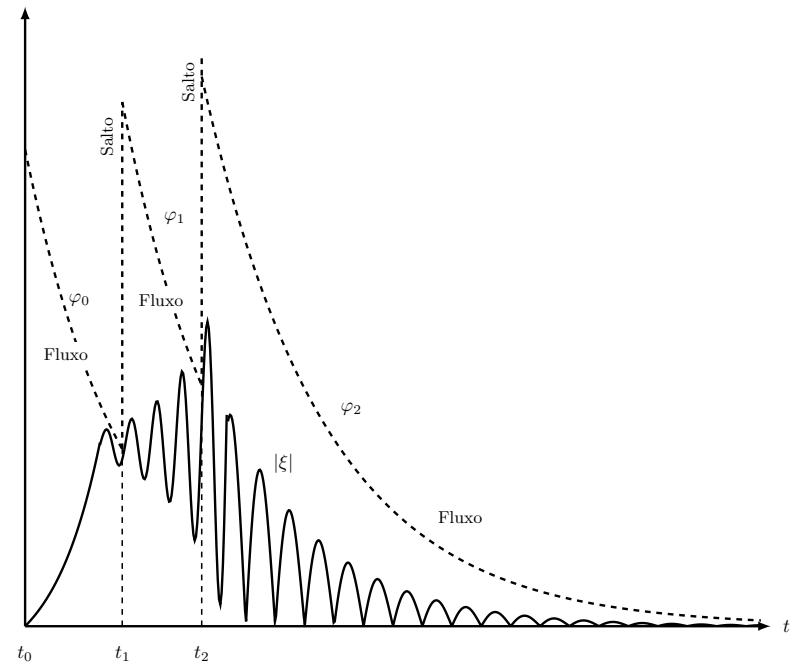

Figura 2. Trajetória da função de monitoração $\varphi_{m}$ (linha pontilhada) e $|\xi(t)|$ (linha sólida).

$$
\begin{aligned}
& \limsup _{t \rightarrow+\infty}\left|\theta(t)-\theta^{*}\right|=\mathcal{O}(\omega+\delta+a) \\
& \limsup _{t \rightarrow+\infty}\left|y(t)-y^{*}\right|=\mathcal{O}\left(\omega^{2}+\delta^{2}+a^{2}\right)
\end{aligned}
$$

independente do sinal da Hessiana.

Prova: Por simplicidade, assumiremos em (9) que o conjunto residual de $\mathcal{O}(\omega+\delta+a)=k_{1}(\omega+\delta+a)$, sendo $k_{1}$ uma constante fixa desconhecida. Considera-se dois casos: $|\xi(t)|>\mathcal{O}(\omega+\delta+a)$ e $|\xi(t)| \leq \mathcal{O}(\omega+\delta+a)$. Enquanto $|\xi(t)|>\mathcal{O}(\omega+\delta+a)$, suponhamos por contradição que a lei de controle $U(t)$, vista em (5), realiza infinitos chaveamentos, $\forall t \in[0,+\infty)$. Então, o termo $a(k)(10)$ aumentará ilimitadamente à medida que $k \rightarrow+\infty$. Assim, existe um valor finito $\kappa>0$ tal que para $k \geq \kappa$ : (i) o termo $a(k)$ será o limitante superior para $k_{1}$ em (9), tal que $k_{1}(\omega+\delta+a)<a(\kappa)(\omega+\delta+a)$, (ii) o termo $R e^{-\lambda_{2} t}<$ $a(\kappa) e^{t / a(\kappa)}$ para $R e^{-\lambda_{2} t}$ em (9), e (iii) o sinal da Hessiana é corretamente estimado, i.e., $(-1)^{(\kappa+m)} \operatorname{sgn}\left(k_{i}\right) \operatorname{sgn}((h \circ$ $\left.l)^{\prime \prime}\left(\theta^{*}\right)\right)=-1$. Por conveniência, define-se

$$
\zeta(t):=e^{-\lambda_{1}\left(t-t_{i}\right)}\left|\xi\left(t_{i}\right)\right|+R e^{-\lambda_{2} t}+\mathcal{O}(\omega+\delta+a),
$$

de acordo com o lado direito da desigualdade (9). A partir do item (i) e (ii), conclui-se que $\varphi_{m}(t)>\zeta(t)$, $\forall t \in\left[t_{\kappa}, t_{\kappa+1}\right)$. Do item (iii), $\zeta$ é o limitante superior válido para $|\xi(t)|$. Consequentemente, nenhum chaveamento irá ocorrer após $t=t_{\kappa}$, i.e., $t_{\kappa+1}=+\infty-$ veja $(12)-\mathrm{o}$ que nos leva a uma contradição. Portanto, $\varphi_{k}(t)$ deve parar o chaveamento após algum valor finito $k=N$ e $t_{N} \in[0,+\infty)$, sempre que $|\xi(t)|>\mathcal{O}(\omega+\delta+a)$. Assim sendo, de (10), (11) e (13), conclui-se que

$$
\begin{gathered}
|\zeta(t)| \leq \underbrace{e^{-\lambda\left(t-t_{N}\right)}\left|\xi\left(t_{N}\right)\right|+a(k) e^{-\frac{t}{a(N)}}+}_{\mathcal{O}(\omega+\delta+a)}, \\
\underbrace{a(N)(\omega+\delta+a)}_{\text {ond }},
\end{gathered}
$$

i.e., o gradiente converge para um conjunto residual de $\mathcal{O}(\omega+\delta+a)$. Lembrando que no caso complementar já te$\operatorname{mos}|\xi(t)| \leq \mathcal{O}(\omega+\delta+a)$. Logo conclui-se que $\xi(t)$ tende localmente para um conjunto residual de $\mathcal{O}(\omega+\delta+a)$ à medida que $t \rightarrow+\infty$. 
A partir de (17), é possível concluir ainda que (14) e (15) são válidas ao menos localmente.

\section{RESULTADOS DE SIMULAÇÃO}

Para o desenvolvimento da simulação, foi o considerado o mapa:

$$
\begin{aligned}
& \dot{x}=\frac{1}{\sqrt{100 \pi}} \exp \left(-\frac{(t-40)^{2}}{100}\right)+\dot{\theta}, \\
& y=-x^{2}+2 x-1 .
\end{aligned}
$$

e os seguintes valores $\omega=35, a=0.1$ e $K=0.1$. Após 150 sgundos, a saída (19) será alterada com o propósito de verificar a efiência do método.

Para encontrar o ponto de convergência da entrada $x(t)$ é necessário derivar a equação da saída (19), obtendo:

$$
\frac{\partial y}{\partial x}=-2 x+2
$$

para, em seguida, igualar a derivada encontrada a zero. Sendo assim, nota-se facilmente que a convergência irá ocorrer em $x=1$. Na Figura 3 é possível acompanhar a entrada $x(t)$ convergindo para o ponto de otimização $x^{*}$.

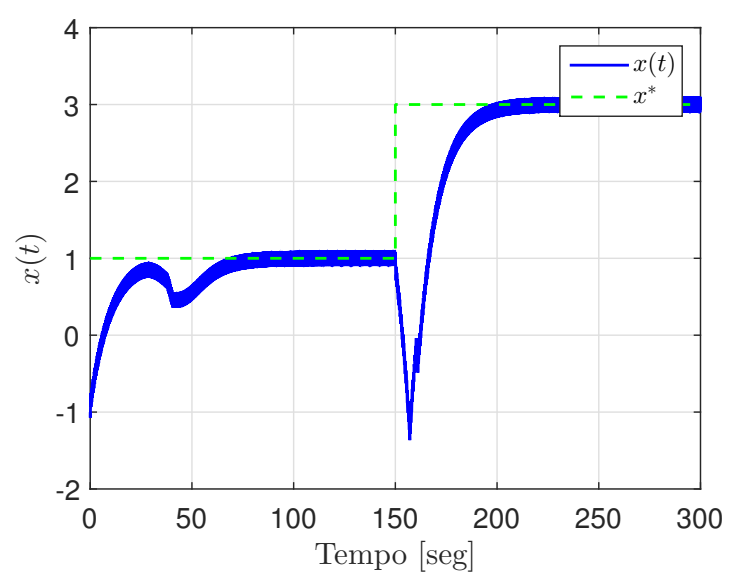

Figura 3. Entrada do mapa $x(t)$ e o ponto de otimização $x^{*}$.

O ponto de convergência da saída $y(t)$ pode ser encontrado substituindo o valor da entrada $x(t)$ em (19). Para esse caso, teremos $y=0$. Na Figura 4 é possível acompanhar a saída $y(t)$ convergindo para o ponto ótimo $y^{*}$.

Por meio de uma derivada de segunda ordem de (19), é possível definir o extremo da função analisada. A partir de

$$
\frac{\partial^{2} y}{\partial x^{2}}=-2
$$

e sendo $\frac{\partial^{2} y}{\partial x^{2}}<0$, trata-se de um ponto de máximo. Ainda analisando a Figura 4, nota-se que o sistema foi inicializado achando se tratar de um problema de mínimo, porém, a saída, $y(t)$, começa a se distanciar do valor ótimo, divergindo em aproximadamente 40 segundos. Visto o erro na interpretação do sistema, a função de monitoração, Figura 5, realiza chaveamentos até a saída seja recuperada e que a convergência do sistema seja garantida, e identificando o extremo como um ponto de máximo.

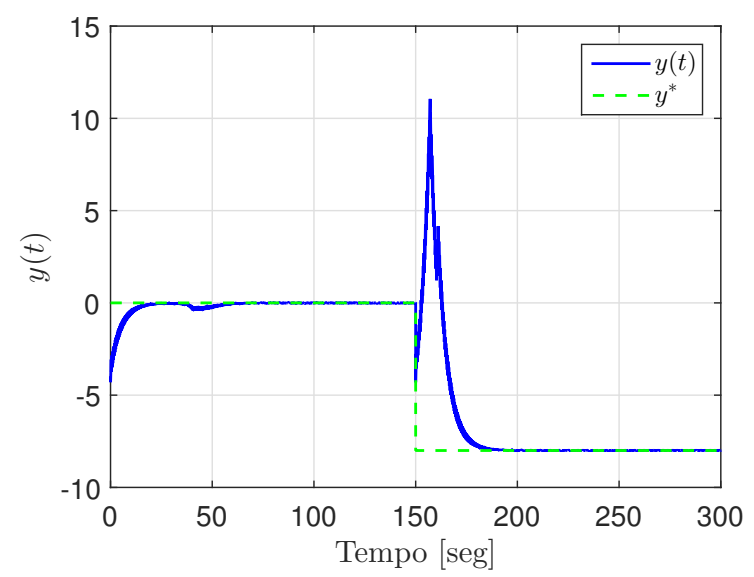

Figura 4. Saída $y(t)$ e valor ótimo $y^{*}$.

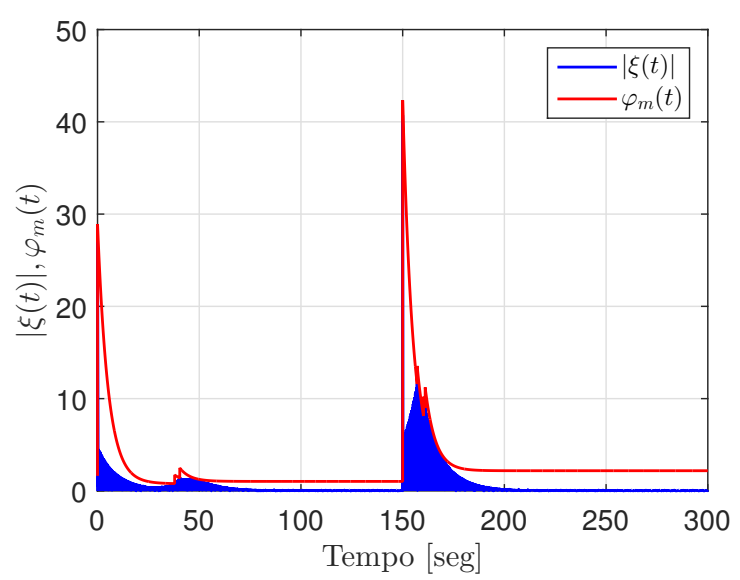

Figura 5. Função de monitoração $\varphi_{m}(t)$ e $|\xi(t)|$.

Com o intuito de testar a eficiência dessa abordagem, após 150 segundos, a equação da saída foi alterada. Para essa mudança de cenário, (18) foi mantida e para $y(t)$ foi definida a seguinte equação

$$
y=x^{2}-6 x+1
$$

Para essa situação, deve ser feita uma nova análise para que se saiba os pontos de convergência de $x$ e $y$. Derivando a equação da saída, temos que

$$
\frac{\partial y}{\partial x}=2 x-6
$$

Ao igualar essa derivada a zero, encontra-se $x=3$ como o ponto de convergência, Figura 3. Substituindo o valor encontrado para $x(t)$ na nova equação de $y(t)$, (22), teremos em $y=-8$, Figura 4 , a convergência da saída. Como na análise anterior, por meio de uma derivada de segunda ordem de (22), será definido o extremo da saída $y(t)$. Sendo

$$
\frac{\partial^{2} y}{\partial x^{2}}=2
$$

com $\frac{\partial^{2} y}{\partial x^{2}}>0$, trata-se de um ponto de mínimo. Analisando novamente a Figura 4, a saída foi interpretada com um sinal de máximo e logo após começa a divergir. Novamente a função de monitoração atua, em aproximadamente 165 
segundos, e seu chaveamento leva a saída a identificar o extremo como mínimo e convergir.

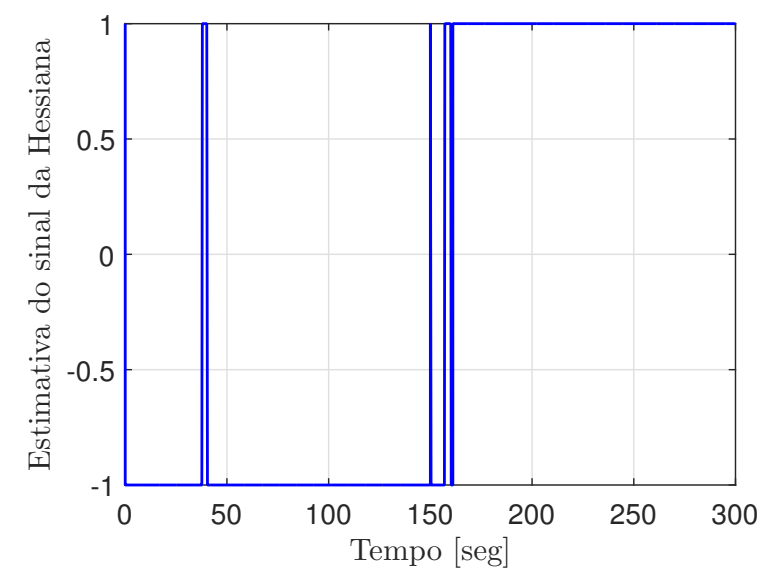

Figura 6. Mudança de sinal da estimativa da Hessiana.

Analisando a Figura 5, observa-se o chaveamento de $\varphi_{m}(t)$, e consequentemente a mudança de sinal da estimativa da Hessiana, Figura 6, ocorre nos momentos em que a saída $y(t)$ começa a se distanciar de seu valor ótimo, e pode se repetir até que garanta a convergência, mesmo que momentânea, tendo um decaimento exponencial da função de monitoração após cada chaveamento.

\section{CONCLUSÕES}

Este artigo, teve como objetivo generalizar o estudo desenvolvido em (Dibo and Oliveira, 2018) e (Dibo and Oliveira, 2019), apresentando uma proposta de otimização em tempo real para o mapeamento dinâmico escalar com sinal de Hessiana desconhecido. Para o desenvolvimento do método, utilizou-se a planta em estudo em (Krstic and Wang, 2000), fazendo as modificações necessárias para considerar o extremo desconhecido. A estratégia escolhida afim contornar esse problema foi a utilização de uma função de monitoração capaz de conduzir o sistema a uma vizinhança do extremo desconhecido. A análise de estabilidade foi realizada e a ideia proposta foi testada em simulações numéricas. A função de monitoração foi capaz de conduzir o sistema à convergência e se mostrou eficiente também quando o ponto de otimização sofre alteração.

\section{REFERÊNCIAS}

Aminde, N.O. (2013). Controle extremal via funções de monitoração e realimentação de saída. Tese de mestrado, COPPE/UFRJ.

Astrom, K.J. and Wittenmark, B. (2005). Adaptive Control. Canada: Addison-Wesley.

Dibo, A. and Oliveira, T.R. (2019). Extremum seeking for static maps with unknown Hessian signs. 27th Mediterranean Conference on Control and Automation, under review, 571-576.

Dibo, A.L. and Oliveira, T.R. (2018). Controle extremal de mapeamentos escalares com sinal de Hessiana desconhecido. XXII Congresso Brasileiro de Automática, $1-7$.
Feiling, J., Koga, S., Krstić, M., and Oliveira, T.R. (2018). Gradient extremum seeking for static maps with actuation dynamics governed by diffusion pdes. Automatica, 95, $197-206$.

Goebel, R., Sanfelice, R.G., and Teel, A. (2012). Hybrid dynamical systems - modeling, stability, and robustness. Princeton University Press., ISBN: 978-0-691-15389-6.

Khalil, H.K. (2002). Nonlinear systems. Englewood Cliffs: Prentice-Hall,.

Krstic, M., Ghaffari, A., and Seshagiri, S. (2014). Extremum seeking for wind and solar energy applications. Proceeding of the 11th World Congress on Intelligent Control and Automation, ISBN: 978-1-4799-5825-2.

Krstic, M. and Wang, H. (2000). Stability of extremum seeking feedback for general nonlinear dynamic systems. Automatica, 36(4), 595-601.

Leblanc, M. (1922). Sur l'electrification des chemins de fer au moyen de courants alternatifs de frequence elevee. Revue Generale de l'Electricite.

Liu, S.J. and Krstic, M. (2012). Stochastic Averaging and Stochastic Extremum Seeking. Springer.

Oliveira, T.R., Krstic, M., and Tsubakino, D. (2017). Extremum seeking for static maps with delays. IEEE Transactions on Automatic Control, 62, 1911 - 1926.

Oliveira, T.R., Peixoto, A.J., Nunes, E.V.L., and Hsu, L. (2007). Control of uncertain nonlinear systems with arbitrary relative degree and unknown control direction using sliding modes. International Journal of Adaptive Control and Signal Processing., 692-707.

Poveda, J.I. and Teel, A.R. (2017). A framework for a class of hybrid extremum seeking controllers with dynamic inclusions. Automatica, 76, $113-126$.

Rodrigues, V.H.P. and Oliveira, T.R. (2017). Global adaptive HOSM differentiators via monitoring functions and hybrid norm-state observers for output feedback. International Journal of Control., DOI:10.1080/00207179.2017.1392041.

Tan, Y., Moase, W., Manzie, C., Nesic, D., and Mareels, I. (2010). Extremum seeking from 1922 to 2010. In: IEEE. Control Conference (CCC), 2010 29th Chinese., 14-26.

Zhang, C. and Ordonez, R. (2012). Extremum Seeking Control and Application: A Numerical OptimizationBased Approach. Springer-VerlagLondom Limited,.

\section{Apêndice A. REVISÃO DO MÉTODO DE BUSCA EXTREMAL CLÁSSICA COM SINAIS DE HESSIANA CONHECIDOS}

Em Krstic and Wang (2000), é considerada a seguinte planta como um modelo não-linear SISO e a seguinte lei de controle:

$$
\begin{aligned}
& \dot{x}=f(t, u), \\
& y=h(x), \\
& u=\alpha(x, \theta) .
\end{aligned}
$$

A partir do sistema são realizadas as seguintes hipóteses sobre o sistema em malha fechada.

Hipótese A.1: Existe uma função suave $l: \mathbb{R} \rightarrow \mathbb{R}^{n}$, tal que:

$$
f(t, \alpha(x, \theta))=0 \quad \text { se e somente se } \quad x=l(\theta) .
$$


Hipótese A.2: Existe $\theta^{*} \in \mathbb{R}$ tal que:

$$
\begin{aligned}
(h \circ l)^{\prime}\left(\theta^{*}\right) & =0, \\
(h \circ l)^{\prime \prime}\left(\theta^{*}\right) & <0,
\end{aligned}
$$

Sintetiza-se, então, o sistema da seguinte forma:

$$
\begin{aligned}
& \dot{x}=f(t, \alpha(x, \hat{\theta}+a \text { sen } \omega t)), \\
& \dot{\hat{\theta}}=k_{i} \xi, \\
& \dot{\xi}=-\omega_{l} \xi+\omega_{l}(y-\eta) a \text { sen } \omega t, \\
& \dot{\eta}=-\omega_{h} \eta+\omega_{h} y . \\
& \tilde{\theta}=\hat{\theta}-\theta^{*}, \\
& \tilde{\eta}=\eta-h \circ l\left(\theta^{*}\right) .
\end{aligned}
$$

O estudo da estabilidade é dividida em etapas, antes de analisar o sistema geral (A.1)-(A.3), fixa-se a variável $x$ em um valor de equilíbrio e é verificada a estabilidade desta pequena parcela. Comprovada a estabilidade desta, partese então para a análise do sistema geral.

$$
\theta=\theta^{*}+\tilde{\theta}+a \operatorname{sen} \tau \text {. }
$$

Tem-se o seguinte sistema reduzido médio:

$$
\begin{aligned}
& \frac{d \tilde{\theta}_{r}}{d \tau}=\delta K^{\prime} \xi_{r}, \\
& \frac{d \xi_{r}}{d \tau}=\delta\left(-\omega_{L}^{\prime} \xi_{r}+\omega_{L}^{\prime}\left(v\left(\tilde{\theta}_{r}+a \operatorname{sen} \tau\right)-\right.\right. \\
& \left.\left.\quad \tilde{\eta}_{r}\right) a \operatorname{sen} \tau\right), \\
& \frac{d \tilde{\eta}_{r}}{d \tau}=\delta\left(-\omega_{H}^{\prime} \tilde{\eta}_{r}+\omega_{H}^{\prime}\left(v\left(\tilde{\theta}_{r}+a \operatorname{sen} \tau\right)\right),\right.
\end{aligned}
$$

Tem-se também que:

$$
\begin{aligned}
v(0) & =0, \\
v^{\prime}(0) & =(h \circ l)^{\prime}\left(\theta^{*}\right)=0, \\
v^{\prime \prime}(0) & =(h \circ l)^{\prime \prime}\left(\theta^{*}\right)<0 .
\end{aligned}
$$

As equações (A.13) a (A.15) estão na forma em que o Teorema da Média Khalil (2002) é aplicado. Seus modelos reduzidos aplicado ao teorema são:

$$
\frac{d}{d \tau}\left[\begin{array}{c}
\tilde{\theta}_{r}^{a} \\
\xi_{r}^{a} \\
\tilde{\eta}_{r}^{a}
\end{array}\right]=\delta\left[\begin{array}{c}
K^{\prime} \xi_{r}^{a} \\
-\omega_{L}^{\prime} \xi_{r}^{a}+\frac{\omega_{L}^{\prime}}{2 \pi} a \int_{0}^{2 \pi} v\left(\tilde{\theta}_{r}^{a}+a \operatorname{sen} \sigma\right) \operatorname{sen} \sigma d \sigma \\
-\omega_{H}^{\prime} \tilde{\eta}_{r}^{a}+\frac{\omega_{H}^{\prime}}{2 \pi} \int_{0}^{2 \pi} v\left(\tilde{\theta}_{r}^{a}+a \operatorname{sen} \sigma\right) d \sigma
\end{array}\right] .
$$

Para linearizar o sistema, é necessário determinar o equilíbrio médio $\left(\tilde{\theta}_{r}^{a, e}, \xi_{r}^{a, e}, \tilde{\eta}_{r}^{a, e}\right)$ que satisfaça as seguintes condições:

$$
\begin{gathered}
\tilde{\xi}_{r}^{a, e}=0, \\
\int_{0}^{2 \pi} v\left(\tilde{\theta}_{r}^{a, e}+a \operatorname{sen} \sigma\right) \operatorname{sen} \sigma d \sigma=0, \\
\tilde{\eta}_{r}^{a, e}=\frac{1}{2 \pi} \int_{0}^{2 \pi} v\left(\tilde{\theta}_{r}^{a, e}+a \operatorname{sen} \sigma\right) d \sigma .
\end{gathered}
$$

Para colocarmos $\tilde{\theta}_{r}^{a, e}$ na forma $\tilde{\theta}_{r}^{a, e}=b_{1} a+b_{2} a^{2}+$ $\mathcal{O}\left(a^{3}\right)$, substituindo em (A.22) as equações (A.17) e (A.18), integrando e igualando as potências de $a$. Teremos, então, $v^{\prime \prime}(0) b_{1}=0$ e $v^{\prime \prime}(0) b_{2}+\frac{1}{8} v^{\prime \prime \prime}(0)=0$. Isso implica em:

$$
\tilde{\theta}_{r}^{a, e}=-\frac{v^{\prime \prime \prime}(0)}{8 v^{\prime \prime}(0)} a^{2}+\mathcal{O}\left(a^{3}\right) .
$$

Aplicando o mesmo raciocínio em (A.22), obtemos:

$$
\tilde{\eta}_{r}^{a, e}=\frac{v^{\prime \prime}(0)}{4} a^{2}+\mathcal{O}\left(a^{3}\right) .
$$

Temos, então, que o equilíbrio do modelo médio é:

$$
\left[\begin{array}{c}
\tilde{\theta}_{r}^{a, e} \\
\xi_{a}^{a, e} \\
\tilde{\eta}_{r}^{a, e}
\end{array}\right]=\left[\begin{array}{c}
-\frac{v^{\prime \prime \prime}(0)}{8 v^{\prime \prime}(0)} a^{2}+\mathcal{O}\left(a^{3}\right) \\
0 \\
\frac{v^{\prime \prime}(0)}{4} a^{2}+\mathcal{O}\left(a^{3}\right)
\end{array}\right] .
$$

Após encontrar a matriz Jacobiana do modelo reduzido, prova-se que a mesma é Hurwitz para um valor suficientemente pequeno de $a$. Com isso, pode-se determinar que os pontos de equillíbrio do sistema médio é exponencialmente estável para um tamanho suficientemente pequeno.

Comprovada a estabilidade do sistema reduzido, os autores voltam ao sistema geral, o reescrevendo na escala de tempo $\tau=\omega t$. A notação compacta é escrita como:

$$
\frac{d z}{d \tau}=\delta G(\tau, x, z)
$$

com isso, por meio da trandormação $\tilde{z}=z+z_{r}^{2 \pi}(\tau)$, leva-se o sistema geral para forma de perturbação padrão singular e obtém-se:

$$
\begin{aligned}
\frac{d \tilde{z}}{d \tau} & =\delta \tilde{G}(\tau, x, \tilde{z}), \\
\omega \frac{d x}{d \tau} & =\tilde{F}(\tau, x, \tilde{z}),
\end{aligned}
$$

onde

$$
\begin{gathered}
\tilde{G}(\tau, x, \tilde{z})=G\left(\tau, x, \tilde{z}+z_{r}^{2 \pi}(\tau)\right)- \\
G\left(\tau, L\left(\tau, z_{r}^{2 \pi}(\tau)\right), z_{r}^{2 \pi}(\tau)\right), \\
\tilde{F}(\tau, x, \tilde{z})=f\left(x, \alpha\left(x, \theta^{*}+\tilde{\theta}-\tilde{\theta}_{r}^{2 \pi}(\tau)+\right.\right. \\
\left.\left.\tilde{\theta}_{r}^{2 \pi}(\tau)+a \operatorname{sen} \tau\right)\right) \\
\frac{d \tilde{z}_{r}}{d \tau}=\delta \tilde{G}\left(\tau, L\left(\tau, \tilde{z}_{r}+z_{r}^{2 \pi}(\tau)\right), \tilde{z}_{r}+z_{r}^{2 \pi}(\tau)\right)
\end{gathered}
$$

tem equilíbrio na origem $\tilde{z}=0$. Para completar a análise da perturbação singular, é necessário estudar também o modelo da camada limite (BLM) na escala de tempo $t-$ $t_{0}=\tau / \omega:$

$$
\begin{aligned}
\frac{d x_{b}}{d t} & =\tilde{F}\left(\tau, x_{b}+L\left(\tau, \tilde{z}+z_{r}^{2 \pi}(\tau)\right), \tilde{z}\right) \\
& =f\left(x_{b}+l(\theta), \alpha\left(x_{b}+l(\theta), \theta\right)\right),
\end{aligned}
$$

Por último, em (Krstic and Wang, 2000), os autores resumem as conclusões acima com os seguintes teoremas:

Teorema A.1: Considere que as Hipóteses A.1 a A.2 sejam satisfeitas. Existe um conjunto de condições iniciais em torno do ponto $(x, \hat{\theta}, \xi, \eta)=\left(l\left(\theta^{*}\right), \theta^{*}, 0, h \circ l\left(\theta^{*}\right)\right)$ e constantes $\bar{\omega}, \bar{\delta}$ e $\bar{a}$ tal que para todo $\omega \in(0, \bar{\omega}), \delta \in(0, \bar{\delta})$ e $a \in(0, \bar{a})$, a solução $(x(t), \hat{\theta}(t), \xi(t), \eta(t))$ converge exponencialmente para uma vizinhança de $\mathcal{O}(\omega+\delta+a)$. Tem-se também que $y(t)$ converge para uma vizinhança de $\mathcal{O}(\omega+\delta+a)$ de $h \circ l\left(\theta^{*}\right)$

Teorema A.2: Dentro das condições do Teorema A.1, existe uma solução periódica exponencialmente estável para uma vizinhança de $\mathcal{O}(\omega+\delta+a)$ do ponto $(x, \hat{\theta}, \xi, \eta)=\left(l\left(\theta^{*}\right), \theta^{*}, 0, h \circ l\left(\theta^{*}\right)\right)$. 